\title{
A note on the Kobayashi pseudodistance and the tautness of holomorphic fiber bundles
}

\author{
by Do Duc Thai and Nguyen Le Huong (Ha noi)
}

\begin{abstract}
We show a relation between the Kobayashi pseudodistance of a holomorphic fiber bundle and the Kobayashi pseudodistance of its base. Moreover, we prove that a holomorphic fiber bundle is taut iff both the fiber and the base are taut.
\end{abstract}

I. Introduction. In this article we shall show a relation between the Kobayashi pseudodistance of a holomorphic fiber bundle and the Kobayashi pseudodistance of its base. Moreover, we shall give another proof (not using universal coverings as in Nag [5]) that a holomorphic fiber bundle is taut iff both the fiber and the base are taut. An analogous result for hyperbolicity was proved by Nag [5].

In this section we recall some definitions and relevant properties.

We denote the Kobayashi pseudodistance on a complex space $M$ by $d_{M}$ (see Kobayashi [2], [3] and Lang [4]). $M$ is called (complete) hyperbolic if $d_{M}$ is a (complete) distance. $M$ is called taut [3] if whenever $N$ is a complex space and $f_{i}: N \rightarrow M$ is a sequence of holomorphic maps, then either there exists a subsequence which is compactly divergent or a subsequence which converges uniformly on compact subsets to a holomorphic map $f: N \rightarrow M$. It suffices that this condition should hold when $N=D[3$, p. 378], where $D$ is the unit disk in $\mathbb{C}$.

Also, a complete hyperbolic space is taut, and a taut complex space is hyperbolic [3, p. 378].

Acknowledgement. The authors are grateful to the referee for many valuable comments.

2. THEOREM A. Let $E$ be a holomorphic fiber bundle over $M$ with hyperbolic fiber $F$ and projection $\pi: E \rightarrow M$, where $E, F, M$ are complex

1991 Mathematics Subject Classification: 32H15, 32L05.

Key words and phrases: holomorphic fiber bundle, Kobayashi pseudodistance, hyperbolic space, taut space. 
manifolds. Then $d_{M}(x, y)=d_{E}\left(\widetilde{x}, \pi^{-1}(y)\right)=\inf _{\tilde{y} \in \pi^{-1}(y)} d_{E}(\widetilde{x}, \widetilde{y})$ for all $x, y \in M$ and all $\widetilde{x} \in \pi^{-1}(x)$.

Pro of. We have $d_{M}(x, y) \leq d_{E}\left(\widetilde{x}, \pi^{-1}(y)\right)$ for all $\widetilde{x} \in \pi^{-1}(x)$. We must prove the reverse inequality. Take arbitrary points $x, y \in M$ and $\widetilde{x} \in \pi^{-1}(x)$. Consider $a_{1}, \ldots, a_{k} \in D$ and $f_{1}, \ldots, f_{k} \in \operatorname{Hol}(D, M)$ such that

$$
f_{1}(0)=x, \quad f_{i}\left(a_{i}\right)=f_{i+1}(0), \quad f_{k}\left(a_{k}\right)=y .
$$

Consider the pull-back diagram

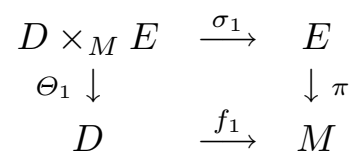

By a result of Royden [6], there is an equivalence $\Phi_{1}: D \times F \rightarrow D \times{ }_{M} E$ of holomorphic fiber bundles over $M$. Thus there exists $c_{1} \in F$ such that $\sigma_{1} \circ \Phi_{1}\left(0, c_{1}\right)=\widetilde{x}$. We define a holomorphic map $\varphi_{1}: D \rightarrow E$ by

$$
\varphi_{1}(z)=\sigma_{1} \circ \Phi_{1}\left(z, c_{1}\right) \quad \text { for all } z \in D .
$$

Consider the pull-back diagram

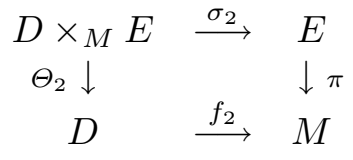

Reasoning as above, there exists an equivalence $\Phi_{2}: D \times F \rightarrow D \times{ }_{M} E$ of holomorphic fiber bundles over $M$ and a point $c_{2} \in F$ such that $\sigma_{2} \circ$ $\Phi_{2}\left(0, c_{2}\right)=\varphi_{1}\left(a_{1}\right)$. Define a holomorphic map $\varphi_{2}: D \rightarrow E$ by

$$
\varphi_{2}(z)=\sigma_{2} \circ \Phi_{2}\left(z, c_{2}\right) \quad \text { for all } z \in D .
$$

Continuing this process we find maps $\varphi_{1}, \ldots, \varphi_{k} \in \operatorname{Hol}(D, E)$ such that $\varphi_{1}(0)=\widetilde{x}, \varphi_{i}\left(a_{i}\right)=\varphi_{i+1}(0), \varphi_{k}\left(a_{k}\right) \in \pi^{-1}(y)$. Thus $d_{M}(x, y) \geq d_{E}\left(\widetilde{x}, \pi^{-1}(y)\right)$ for all $\widetilde{x} \in \pi^{-1}(x)$.

3. Lemma. Let $\pi: \widetilde{X} \rightarrow X$ be a holomorphic map between two complex spaces satisfying the following: For every $x \in X$ there exists an open neighbourhood $U_{x}$ of $x$ such that $\pi^{-1}\left(U_{x}\right)$ is taut. Then $\widetilde{X}$ is taut if so is $X$.

Proof. Assume that $X$ is taut and a sequence $\left\{\widetilde{f}_{n}\right\} \subset \operatorname{Hol}(D, \widetilde{X})$ is not compactly divergent. Without loss of generality we may suppose that there exists a sequence $\left\{z_{n}\right\} \subset D$ converging to a point $z \in D$ such that $\left\{\widetilde{f}_{n}\left(z_{n}\right)\right\}$ converges to a point $\widetilde{p} \in \widetilde{X}$.

Obviously $\left\{f_{n}=\pi \circ \widetilde{f}_{n}\right\} \subset \operatorname{Hol}(D, X)$ is not compactly divergent and we can assume that $\left\{f_{n}\right\}$ converges uniformly to a map $F \in \operatorname{Hol}(D, X)$. 
Put $p=\pi(\widetilde{p})=F(z)$. Take an open neighbourhood $U_{p}$ of $p$ such that $\pi^{-1}\left(U_{p}\right)$ is taut. Since $\left\{f_{n}\right\}$ converges to $F$, there exists an open subset $V$ of $D$ such that $f_{n}(V) \subset U_{p}$ for all $n \geq N$.

On the other hand, since $\left\{\widetilde{f}_{n}\left(z_{n}\right)\right\}$ converges to $\widetilde{p}$ and since $\pi^{-1}\left(U_{p}\right)$ is taut, we can assume that $\left\{\widetilde{f}_{n} \mid V\right\} \subset \operatorname{Hol}\left(V, \pi^{-1}\left(U_{p}\right)\right)$ converges uniformly to a map $\widetilde{F} \in \operatorname{Hol}(V, \widetilde{X})$.

Consider the family $\mathcal{V}$ of all pairs $(W, \Phi)$, where $W$ is an open subset of $D$ and $\Phi \in \operatorname{Hol}(W, \widetilde{X})$ such that there exists a subsequence $\left\{\widetilde{f}_{n_{k}} \mid W\right\}$ of $\left\{\widetilde{f}_{n} \mid W\right\}$ which converges uniformly to $\Phi$ in $\operatorname{Hol}(W, \widetilde{X})$.

We define an order relation in $\mathcal{V}$ by setting $\left(W_{1}, \Phi_{1}\right) \leq\left(W_{2}, \Phi_{2}\right)$ if $W_{1} \subset$ $W_{2}$ and for every subsequence $\left\{\widetilde{f}_{n_{k}} \mid W_{1}\right\}$ of $\left\{\widetilde{f}_{n} \mid W_{1}\right\}$ converging to $\Phi_{1}$ in $\operatorname{Hol}\left(W_{1}, \widetilde{X}\right)$ uniformly on compact sets, the sequence $\left\{\widetilde{f}_{n_{k}} \mid W_{2}\right\}$ contains a subsequence converging to $\Phi_{2}$ in $\operatorname{Hol}\left(W_{2}, \widetilde{X}\right)$. Assume that $\left\{\left(W_{\alpha}, \Phi_{\alpha}\right)\right\}_{\alpha \in \Lambda}$ is a well-ordered subset of $\mathcal{V}$. Put $W_{0}=\bigcup_{\alpha \in \Lambda} W_{\alpha}$. Define $\Phi_{0} \in \operatorname{Hol}\left(W_{0}, \widetilde{X}\right)$ by $\Phi_{0} \mid W_{\alpha}=\Phi_{\alpha}$ for all $\alpha \in \Lambda$. Take a sequence $\left\{\left(W_{i}, \Phi_{i}\right)\right\}_{i=1}^{\infty} \subset\left\{\left(W_{\alpha}, \Phi_{\alpha}\right)\right\}_{\alpha \in \Lambda}$ such that $\left(W_{1}, \Phi_{1}\right) \leq\left(W_{2}, \Phi_{2}\right) \leq \ldots$ and $W_{0}=\bigcup_{i=1}^{\infty} W_{i}$. Then there exists a subsequence $\left\{\widetilde{f}_{n}^{1} \mid W_{1}\right\}$ of $\left\{\widetilde{f}_{n} \mid W_{1}\right\}$ converging to $\Phi_{1}$ in $\operatorname{Hol}\left(W_{1}, \widetilde{X}\right)$.

Consider $\left\{\widetilde{f}_{n}^{1} \mid W_{2}\right\}$. As above $\left\{\widetilde{f}_{n}^{1}\right\}$ contains a subsequence $\left\{\widetilde{f}_{n}^{2}\right\}$ such that $\left\{\widetilde{f}_{n}^{2} \mid W_{2}\right\}$ converges to $\Phi_{2}$ in $\operatorname{Hol}\left(W_{2}, \widetilde{X}\right)$.

Continuing this process we get sequences $\left\{\widetilde{f}_{n}^{k}\right\}$ such that $\left\{\widetilde{f}_{n}^{k}\right\} \in\left\{\widetilde{f}_{n}^{k-1}\right\}$ for every $k \geq 2$ and $\left\{\widetilde{f}_{n}^{k} \mid W_{k}\right\}$ converges uniformly to $\Phi_{k}$ in $\operatorname{Hol}\left(W_{k}, \widetilde{X}\right)$. Therefore $\left\{\widetilde{f}_{k}^{k}\right\}$ converges to $\Phi_{0}$ in $\operatorname{Hol}\left(W_{0}, \widetilde{X}\right)$.

Thus $\left(W_{0}, \Phi_{0}\right) \in \mathcal{V}$ and hence the subset $\left\{\left(W_{\alpha}, \Phi_{\alpha}\right)\right\}_{\alpha \in \Lambda}$ has an upper bound. By Zorn's Lemma, the family $\mathcal{V}$ has a maximal element $(W, \Phi)$.

Let $\left\{\widetilde{f}_{n_{k}} \mid W\right\}$ be a subsequence of $\left\{\widetilde{f}_{n} \mid W\right\}$ converging uniformly to $\Phi$ in $\operatorname{Hol}(W, \widetilde{X})$. Take $z \in \bar{W}$ and an open neighbourhood $U$ of $F\left(z_{0}\right)$ in $X$ such that $\pi^{-1}(U)$ is taut. Since $\left\{f_{n}\right\}$ converges uniformly to $F \in \operatorname{Hol}(D, X)$ in $\operatorname{Hol}(D, X)$, there exists an open neighbourhood $W_{0}$ of $z_{0}$ in $D$ such that $\left(\pi \circ \widetilde{f}_{n}\right)\left(W_{0}\right) \subset U$ for all $n \geq 1$. Hence $\widetilde{f}_{n}\left(W_{0}\right) \subset \pi^{-1}(U)$ for all $n \geq 1$.

Take $z_{1} \in W_{0} \cap W$. Then $\left\{\widetilde{f}_{n_{k}}\left(z_{1}\right)\right\}$ is convergent. By the normality of the family $\operatorname{Hol}\left(W_{0}, \pi^{-1}(U)\right)$ and by the maximality of $(W, \Phi)$ we have $W_{0} \subset W$ and $W=D$.

Notice that for the hyperbolicity the above lemma was proved by Eastwood [1].

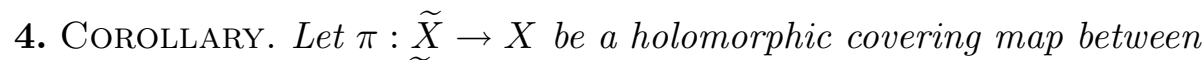
two complex spaces. Then $\widetilde{X}$ is taut if and only if so is $X$.

Proof. $\Rightarrow$ Assume that $\widetilde{X}$ is taut and a sequence $\left\{f_{n}\right\} \subset \operatorname{Hol}(D, X)$ is not compactly divergent. Then there exists a sequence $\left\{z_{n}\right\} \subset D$ converging 
to $z_{0} \in D$ such that $\left\{f_{n}\left(z_{n}\right)\right\}$ converges to $p \in X$. Put $f_{n}\left(z_{n}\right)=y_{n}$.

Since $\widetilde{X}$ is taut, $X$ is hyperbolic. Take $\widetilde{p} \in \pi^{-1}(p)$. Since $d_{X}\left(y_{n}, p\right)=$ $\inf _{\tilde{y}_{n} \in \pi^{-1}\left(y_{n}\right)} d_{\tilde{X}}\left(\widetilde{y}_{n}, \widetilde{p}\right)$ (see [2]), there exists $\widetilde{y}_{n} \in \pi^{-1}\left(y_{n}\right)$ such that $d_{\tilde{X}}\left(\widetilde{y}_{n}, \widetilde{p}\right)<d_{X}\left(y_{n}, p\right)+1 / n$. Lift $f_{n}$ to a map $\widetilde{f}_{n}$ such that $\pi \circ \widetilde{f}_{n}=f_{n}$ and $\widetilde{f}_{n}\left(z_{n}\right)=\widetilde{y}_{n}$. Since $\left\{\widetilde{f}_{n}\left(z_{n}\right)\right\}$ converges to $\widetilde{p}$ and $\widetilde{X}$ is taut, we can assume that $\left\{\widetilde{f}_{n}\right\}$ converges uniformly to a map $\widetilde{f} \in \operatorname{Hol}(D, \widetilde{X})$ in $\operatorname{Hol}(D, \widetilde{X})$. Then $\left\{f_{n}\right\}$ converges to $\pi \circ \widetilde{f}$ in $\operatorname{Hol}(D, X)$.

$\Leftarrow$ The proof is deduced immediately from Lemma 3 .

5. TheOREM B. Let $E$ be a holomorphic fiber bundle over $M$ with fiber $F$ and projection $\pi: E \rightarrow M$, where $E, F, M$ are complex manifolds. Then $E$ is taut if and only if both $F$ and $M$ are.

Pro of. $\Rightarrow$ Assume that $E$ is taut. Since $F$ embeds as a closed complex subspace in $E, F$ is taut. Assume that a sequence $\left\{f_{n}\right\} \subset \operatorname{Hol}(D, M)$ is not compactly divergent. Without loss of generality we may suppose that there exists a sequence $\left\{z_{n}\right\} \subset D$ converging to $z \in D$ such that $\left\{y_{n}=\right.$ $\left.f_{n}\left(z_{n}\right)\right\}$ converges to $p \in M$. Take $\widetilde{p} \in \pi^{-1}(p)$. By Theorem A, $d_{M}\left(y_{n}, p\right)=$ $\inf _{x_{n} \in \pi^{-1}\left(y_{n}\right)} d_{E}\left(\widetilde{p}, x_{n}\right)$. Hence there exists $x_{n} \in E$ such that $d_{E}\left(\widetilde{p}, x_{n}\right)<$ $d_{M}\left(p, y_{n}\right)+1 / n$.

Pull back the fiber bundle $\pi: E \rightarrow M$ to a fiber bundle $\Theta_{n}: D \times_{M} E \rightarrow$ $D$ getting a commutative diagram

$$
\begin{array}{cll}
D \times_{M} E & \stackrel{\sigma_{n}}{\longrightarrow} & E \\
\Theta_{n} \downarrow & & \downarrow \pi \\
D & \stackrel{f_{n}}{\longrightarrow} & M
\end{array}
$$

As in the proof of Theorem A there exists an equivalence $\Phi_{n}: D \times F \rightarrow$ $D \times_{M} E$ of holomorphic fiber bundles over $D$ and $c_{n} \in F$ such that $\sigma_{n}$ 。 $\Phi_{n}\left(z_{n}, c_{n}\right)=x_{n}$. Put $\varphi_{n}(z)=\sigma_{n} \circ \Phi_{n}\left(z, c_{n}\right)$ for all $z \in D$. It is easy to see that $\left\{\varphi_{n}\left(z_{n}\right)\right\}$ converges to $\widetilde{p} \in E$. Without loss of generality we may assume that $\left\{\varphi_{n}\right\}$ converges to a map $\varphi \in \operatorname{Hol}(D, E)$ in $\operatorname{Hol}(D, E)$. Hence $\left\{f_{n}\right\}$ converges to $\pi \circ \varphi$ in $\operatorname{Hol}(D, M)$.

$\Leftarrow$ The converse is deduced immediately from Lemma 3 .

\section{References}

[1] A. Eastwood, A propos des variétés hyperboliques complètes, C. R. Acad. Sci. Paris 280 (1975), 1071-1075.

[2] S. Kobayashi, Hyperbolic Manifolds and Holomorphic Mappings, Dekker, New York 1970.

[3] -, Intrinsic distance, measures and geometric function theory, Bull. Amer. Math. Soc. 82 (1976), 357-416.

[4] S. Lang, Introduction to Hyperbolic Complex Spaces, Springer, 1987. 
[5] S. Nag, Hyperbolic manifolds admitting holomorphic fiberings, Bull. Austral. Math. Soc. 26 (1982), 181-184.

[6] H. L. Royden, Holomorphic fiber bundles with hyperbolic fiber, Proc. Amer. Math. Soc. 43 (1974), 311-312.

DEPARTMENT OF MATHEMATICS

PEDAGOGICAL INSTITUTE NO. 1 OF HA NOI

HA NOI, VIET NAM

Reçu par la Rédaction le 9.5.1990

Révisé le 1.9.1990 et 5.1.1991 